\title{
Formación docente en educación para la paz, en la Escuela Normal Superior del municipio de La Cruz - Nariño ${ }^{1}$
}

\author{
Diego Andrés Quintero Timaná ${ }^{2}$ \\ Universidad Mariana, Colombia
}

Recepción: 19/02/2020

Evaluación: 26/02/2020

Aprobación: 09/04/2020

Artículo de Investigación-Reflexión

DOI: https://doi.org/10.22267/rhec.202424.73

\section{Resumen}

En este artículo, se analizan los procesos de formación docente en educación para la paz que se desarrollan en el ciclo de formación complementaria de la Escuela Normal Superior, del municipio de La Cruz, Departamento de Nariño. Para ello, se caracterizan las dinámicas socioeconómicas de la población estudiantil; posteriormente, se identifican las estrategias de enseñanza y aprendizaje que se implementan en el ciclo de formación complementaria en materia de educación para la paz y, por último, se proponen ejes de fortalecimiento en la formación de docentes normalistas en torno a la construcción de una cultura de paz, a partir del ejercicio docente. Así, a través de una estrategia metodológica cualitativa, y mediante el estudio de caso del municipio de La Cruz, se logran examinar los principios pedagógicos sobre los cuales se fundamenta la formación de docentes normalistas y se proponen estrategias que posibilitarán a los maestros atender de forma oportuna las necesidades pedagógicas en materia de resolución de conflictos, convivencia escolar, reconciliación y participación estudiantil, consecuente con el entorno que los rodea.

1 Este artículo de investigación se deriva del proyecto: “Competencias docentes en educación para la paz, en el ciclo de formación complementaria de la Escuela Normal Superior de La Cruz - Nariño".

2 Profesor tiempo completo de la Universidad Mariana, Colombia. Licenciado en Ciencias Sociales y Especialista en Estudios Latinoamericanos por la Universidad de Nariño. Magíster en Sociología por la Facultad Latinoamericana de Ciencias Sociales FLACSO, Ecuador. Grupo de investigación: EduMultiverso. Lineas de investigación: Estudios regionales. Correo electrónico: Diegoaqt92@gmail.com. 나ttp://orcid.org/0000-0001-6631-1969 
Palabras clave: competencias docentes; convivencia escolar; educación para la paz; formación de maestros.

\title{
Teacher training in education for peace, in Escuela Normal Superior La Cruz - Nariño
}

\begin{abstract}
This article aims to analyze the teacher training in education for the peace process that is developed in the Escuela Normal Superior, in the municipality of La Cruz, Department of Nariño. To do so, the socioeconomic dynamics of the student population are characterized; subsequently, the teaching and learning strategies that are implemented in the complementary training cycle in the area of education for peace are identified and, finally, the strengthening of the training of teachers in the construction of a culture of peace is proposed, based on the teaching practice. Thus, through a qualitative methodological strategy, and through the case study of the municipality of La Cruz, it was possible to examine the pedagogical principles on which the training of teacher is based and to propose strategies that will enable teachers to help students in their pedagogical needs in terms of conflict resolution, school coexistence, reconciliation and student participation, consistent with the environment that surrounds them.
\end{abstract}

Keywords: teaching competencies; school coexistence; peace education, teacher training.

\section{Formação de professores em educação para a paz, na Escola Normal Superior do município de La Cruz - Nariño}

\section{Resumo}

Neste artigo, são analisados os processos de formação de professores em educação para a paz que se desenvolvem no ciclo de formação complementar da Escola Normal Superior, do município de la Cruz, Departamento de 
Nariño. Para isso, caracteriza-se a dinâmica socioeconômica da população estudantil; Posteriormente, são identificadas as estratégias de ensino e aprendizagem que se concretizam no ciclo complementar de formação na área da educação para a paz e, por fim, propõem-se eixos no fortalecimento na formação de professores do ensino normal em torno da construção de uma cultura. de paz, desde o exercício docente. Assim, através de uma estratégia metodológica qualitativa, e através do estudo de caso do município de La Cruz, é possível examinar os princípios pedagógicos em que se baseia a formação de professores do ensino normal e são propostas estratégias que permitirão aos professores assistir de forma oportuna necessidades pedagógicas em termos de resolução de conflitos, convivência escolar, conciliação e participação dos alunos, compatíveis com o ambiente que os rodeia.

Palavras chave: habilidades docentes; convivência escolar; educação para a paz; formação de professores.

\section{Introducción}

El municipio de La Cruz se encuentra ubicado en la cordillera andina, a una distancia de $103 \mathrm{~km}$ de la ciudad de San Juan de Pasto, al nororiente del Departamento de Nariño. Este territorio ha sido impactado por las secuelas del conflicto armado en Colombia, a través de los enfrentamientos entre grupos armados al margen de la ley y las fuerzas militares del Estado, los elevados índices de producción y comercialización de cultivos ilícitos, y la baja capacidad institucional en materia de cobertura en salud, educación, vías de comunicación, empleo y vivienda. Tenemos, entonces, que esta región se caracteriza como un territorio con un alto índice de necesidades básicas insatisfechas, violencia y vulnerabilidad, entre los más de dieciocho mil habitantes que conforman aproximadamente su núcleo poblacional.

En este contexto, se encuentra ubicada la Escuela Normal Superior del Mayo, como una institución de carácter público, que presta sus servicios educativos en los niveles de preescolar, básica primaria, bachillerato y el ciclo de formación complementaria, que tiene como objetivo la formación de maestros, según se estipula en la Ley general de educación, Ley 115, de febrero 8 de 1994. El programa de formación complementaria forma docentes desde principios pedagógicos como la educabilidad, la 
enseñabilidad, la pedagogía y la relación con el contexto, tal como se estipula en su Proyecto Educativo Institucional. La Ley General de Educación, en su Artículo 12, establece que a las Escuelas Normales Superiores se las autoriza para formar educadores en el nivel de preescolar y en el ciclo de educación básica y operar como unidades de apoyo académico para la formación inicial de docentes, y podrán ofrecer formación complementaria que conduzca al otorgamiento del título de normalista superior.

Así mismo, cabe señalar que, hacia el año 2015, se promulgó, en Colombia, la Ley 1732 y el Decreto 1038, mediante los cuales se reglamentó la implementación de la Cátedra de la Paz en los establecimientos educativos del país. Entre sus objetivos, se encuentra fomentar el proceso de apropiación de conocimientos y competencias relacionadas con el territorio, la cultura, el contexto económico y social y la memoria histórica, con el propósito de reconstruir el tejido social, promover la prosperidad y garantizar la efectividad de los principios, derechos y deberes consagrados en la Constitución, según lo estipulado en el Decreto 1038, de 2015.

Ante lo expuesto, en este artículo, se analizan los procesos de formación en educación para la paz que se desarrollan en el ciclo de formación complementaria de la Escuela Normal Superior de La Cruz, al considerar que la implementación de la Cátedra de Paz requiere unas competencias específicas por parte del docente para el tratamiento pedagógico de los componentes que la integran y tener en cuenta las dinámicas específicas que el conflicto tuvo en los territorios, para así responder de forma oportuna a las necesidades de educar en la reconciliación, la convivencia, el respeto por las diferencias, la resolución pacífica de conflictos y las necesidades regionales en materia de construcción y promoción de una cultura de paz, especialmente en escenarios educativos que, al igual que en el municipio de La Cruz, fueron impactados por el conflicto armado vivido en Colombia.

Entre los referentes conceptuales, se retoman algunos de los planteamientos que conciben las competencias como "las capacidades que tienen los individuos para interrelacionar sus habilidades cognitivas e intelectuales, con aquellas psicomotrices y afectivas en los escenarios de la vida social". ${ }^{3}$ Además, estos factores permiten delimitar el concepto de las

3 Análida Pinilla, Reflexiones en educación universitaria (Bogotá: Unibiblios, 1999), 114. 
competencias como "el conjunto de habilidades, conocimientos, aptitudes, actitudes y valores que permiten al ser humano relacionarse con el mundo". ${ }^{4}$

Por su parte, en el Decreto 4790, de diciembre de 2008, se establecen las condiciones básicas de calidad de los programas de formación complementaria, en la que el docente normalista se caracteriza por una sólida formación en teorías educativas, pedagógicas y didácticas, así como, también, por la comprensión analítica y reflexiva acerca de los factores inherentes a los procesos diferenciales de la enseñanza y aprendizaje, que les permite profundizar en diferentes alternativas pedagógicas y didácticas para el aprendizaje de los estudiantes.

En este sentido, se examinan las interrelaciones que se presentan entre los procesos de formación de los maestros normalistas y las competencias específicas que se requieren para dinamizar los contenidos propuestos en la Cátedra de Paz, cuyo objetivo se centra en "fomentar el proceso de apropiación de conocimientos y competencias relacionados con el territorio, la cultura, el contexto económico y social y la memoria histórica, para la reconstrucción el tejido social, promover la prosperidad general y garantizar la efectividad de los principios, derechos y deberes consagrados en la Constitución". ${ }^{5}$

A partir del análisis de la literatura académica ${ }^{6}$ que gira en torno a la temática de estudio, se detallan los principales referentes conceptuales desde los cuales se puede analizar el tipo de competencias que se requieren fortalecer en la formación de docentes normalistas para optimizar la implementación de la cátedra de paz en las instituciones educativas del país. Examinar estos referentes posibilitará a los docentes y a las instituciones educativas afrontar las problemáticas de violencia en los contextos escolares, que han germinado de una cultura de la violencia, incidida por los más de cincuenta años de conflicto armado en Colombia. Por ello, el mejoramiento

4 Jerome Bruner, La educación, puerta de la cultura (Madrid: Visor, 1997), 171.

5 Decreto 1038, que reglamenta la cátedra de paz (Bogotá: Presidencia de la República, 2015).

6 Sobre la temática ver Mora-García, José. "Aproximación a las Pedagogías Alternativas: de la Pedagogía de la Diversidad a las Pedagogías de la Resiliencia en el Marco del Postacuerdo". Revista Historia de la Educación Colombiana. vol 22 No 22, (2019): 39-66. https://doi.org/10.22267/rhec.192222.51. Restrepo Mesa, Karol. "Propuesta de Formación del Maestro-Mediador para la Consolidación de la Cultura de Paz en Colombia". Revista Historia de la Educación Colombiana. vol 22 No 22, (2019): 67-91. https://doi.org/10.22267/rhec.192222.52. 
de capacidades, como educar en la no violencia, el mejoramiento de la convivencia en los contextos educativos y la resolución pacífica de conflictos, se convierten en los pilares fundamentales que contribuyen a configurar territorios de paz, mediante el fortalecimiento de la educación y la formación de maestros en territorios que presentaron una alta incidencia del conflicto armado.

\section{Metodología}

Dadas sus características, este artículo es el resultado de un proceso investigativo que se configuró desde un enfoque cualitativo, en tanto se enfoca en comprender un proceso social a partir de las perspectivas de los participantes en relación con su contexto. En concordancia con los objetivos propuestos, se desarrolló como método de investigación el estudio de caso, en tanto se caracteriza por ser un método dirigido a la comprensión de las dinámicas presentadas en contextos particulares, que incita a la reflexión y, al mismo tiempo, genera un conocimiento que permite la comprensión de casos similares en otros contextos.

Para la selección de la muestra de análisis, se consideró que la Escuela Normal Superior de La Cruz, en el año 2018, ofrecía sus servicios educativos en preescolar, básica primaria y bachillerato a 1100 estudiantes, mientras que 65 se encuentran matriculados en el ciclo de formación complementaria, de los cuales se tomó una muestra poblacional intencional de 35 estudiantes, quienes conforman el curso de primer semestre, además de cinco docentes del ciclo de formación complementaria de la institución, para la aplicación de la encuesta y entrevista que permitieron indagar en información para el desarrollo de los objetivos propuestos.

La muestra poblacional analizada se seleccionó mediante un tipo de muestreo no probabilístico; es decir, no se utilizó un procedimiento aleatorio en la selección de los individuos, sino, por el contrario, los individuos se seleccionaron a partir de ciertas características, fijadas en el proceso de investigación. Se empleó un "muestreo de expertos", desde el cual se recurre a la opinión o valoración de sujetos expertos en el tema de estudio, lo que posibilita generar una información más precisa y detallada acerca del fenómeno y las variables de estudio.

Se establecieron, como técnicas de acopio de información, el análisis de contenido, la entrevista semiestructurada y la encuesta sociodemográfica. 
La implementación de estas técnicas posibilitó obtener información referente a los tipos de competencias docentes en educación para la paz que se desarrollan y se requieren fortalecer en la educación de maestros del ciclo de formación complementaria de la Escuela Normal Superior del municipio de La Cruz. A través del análisis de contenido, se logró examinar de manera sistemática el contenido manifiesto en diferentes fuentes de información, tales como recursos bibliográficos relacionados con el establecimiento de la cátedra de paz en Colombia, así como, también, en documentos relacionados con el fortalecimiento de competencias docentes en educación para la paz.

Por su parte, la entrevista semiestructurada permitió encuentros dirigidos hacia la comprensión de la temática de estudio, desde las perspectivas de los docentes y directivos de la institución. La implementación de una encuesta sociodemográfica tuvo como objetivo conocer o describir las características de la población en estudio, a partir de elementos como su zona de residencia, su estrato socioeconómico, el nivel de escolaridad de los padres, el nivel de ingresos económicos del grupo familiar, que contribuyen, entre otros aspectos, a la comprensión de las características socioeconómicas que distinguen a los maestros normalistas en formación del municipio de La Cruz.

\section{Planteamiento del problema}

El municipio de La Cruz ha sido un territorio marcado por las secuelas del conflicto armado en la población civil. Episodios como los constantes hostigamientos a la población por parte de grupos armados y presencia de cultivos ilícitos en las áreas rurales, lo caracterizan como un territorio con una alta incidencia del conflicto armado y una baja capacidad institucional del Estado ante los requerimientos y aspiraciones de más dieciocho mil habitantes, que conforman aproximadamente su núcleo poblacional.

En este contexto, en la cabecera municipal se encuentra ubicada la Escuela Normal Superior de La Cruz, como una institución educativa de carácter público y acreditada por el Ministerio de Educación Nacional, que presta sus servicios educativos en educación básica y media, así como, también, el ciclo de formación complementaria para la preparación de maestros en el nivel de preescolar y primaria, según los lineamientos estipulados por la Ley general de educación, Ley 115, de febrero 8 de 1994. 
El programa de formación complementaria, que se ofrece en la Escuela Normal Superior de La Cruz, forma a docentes desde principios pedagógicos como la educabilidad, la enseñabilidad, la pedagogía y la relación con el contexto, tal como se estipulan en su Proyecto Educativo Institucional. Sin embargo, aunque si bien, en la Ley 1732 y en el Decreto 1038, se plantea la implementación de la Cátedra por la paz en los Planes de estudio de las instituciones educativas del país, esta propuesta de investigación pretende abordar el estudio de las competencias docentes que se requieren para la enseñanza de la Cátedra por la paz tanto de los docentes como de los maestros en formación que se desempeñarán en contextos sociales marcados por las secuelas del conflicto armado, como el municipio de La Cruz, en el Departamento de Nariño. ${ }^{7}$

Por tanto, para afianzar los principios pedagógicos sobre los cuales se fundamenta la formación de docentes normalistas, es pertinente realizar un diagnóstico de las estrategias de enseñanza y aprendizaje que se implementan en el ciclo de formación complementaria, para, posteriormente, establecer las áreas de fortalecimiento en materia de competencias docentes en educación para la paz, que posibilitarán a los estudiantes atender de forma oportuna las necesidades pedagógicas en materia de derechos humanos, resolución de conflictos, prevención del acoso escolar y participación ciudadana, en los escenarios educativos en los cuales se desempeñen como docentes.

De esta manera, se requiere integrar a los estudiantes, docentes y coordinadores del ciclo de formación complementaria de la Escuela Normal Superior de La Cruz, para establecer una serie de ejes de fortalecimiento de las capacidades docentes, que respondan a las necesidades de educar en la tolerancia y reconciliación, en la resolución de conflictos, la convivencia y el fortalecimiento del tejido social, consecuente con el entorno y las necesidades regionales en materia de construcción y promoción de una cultura de paz; así como, también, para generar lineamientos que fortalezcan las instituciones educativas del Estado encargadas de la formación de maestros, como las Escuelas Normales Superiores, y que sus experiencias y contribuciones se replicasen en otros escenarios educativos a los que, al igual que La Cruz, los impactó el conflicto armado vivido en Colombia.

7 Ver: Muñoz Bravo, Jose. "Las Escuelas Normales, orientan la Formación de maestros superiores, comprometidas con la educación y la Práctica Pedagógica Investigativa". Revista Historia de la Educación Colombiana Vol. 23 No 23 (2019), 139-171. https:// doi.org/10.22267/rhec.192323.62. 


\section{Características socioeconómicas de la población estudiantil del ciclo de formación complementaria en la Escuela Normal del municipio de La Cruz-Nariño}

Para identificar las características sociodemográficas de la población estudiantil del ciclo de formación complementaria, a partir de los elementos en común que caracterizan a los estudiantes, se realizó una encuesta a una muestra poblacional de 35 estudiantes, para indagar en torno a sus características sociales, económicas, educativas, su proyecto de vida y percepción sobre la incidencia de la educación para la paz, en su desempeño como futuros docentes.

En la muestra poblacional, encontramos una división del 50\% entre hombres y mujeres; el $69 \%$ de los encuestados se encuentra en un rango de edad entre los 18 y 24 años. El $72 \%$ de la población es soltera y el $82 \%$ reside en el área urbana del municipio de La Cruz. Así mismo, es importante señalar que, según los indicadores del Departamento Nacional de Planeación y del Departamento Administrativo Nacional de Estadística, en Colombia se clasifica a la población en condición de pobreza, en tanto sus ingresos económicos no cubren el costo per cápita mínimo necesario por persona que, en el año 2017, fue de $\$ 250.620$, para las zonas urbanas, y de $\$ 165.062$, para las zonas rurales.

Esta dinámica económica permite establecer que, si bien el $75 \%$ de los estudiantes encuestados compone un núcleo familiar de entre 1 a 5 personas, los ingresos económicos mensuales familiares del 90,6\% no superan el salario mínimo vigente, por lo cual se puede caracterizar a un elevado porcentaje de los encuestados como una población en situación de precariedad económica. Así mismo, el 41\% de los encuestados reside en una vivienda en arriendo, mientras que el $34,4 \%$ reside en una vivienda propia y el $21,9 \%$ en una vivienda familiar.

En un primer momento, a la población estudiantil del ciclo de formación complementaria se le indagó en torno a sus proyectos de vida, concebidos desde su etapa escolar de bachillerato. Los resultados de la encuesta muestran que un $47 \%$ de los estudiantes tenía como propósito el estudio de una carrera universitaria, mientras que el $35 \%$ se proyectaba en ingresar al ciclo de formación complementaria. Estos dos componentes representan los mayores porcentajes entre los encuestados, elementos que nos llevan a tener en cuenta el deseo de continuar con sus estudios posteriores a la 
etapa de bachillerato, aunque si bien el mayor porcentaje de la población se proyectaba en una carrera universitaria, por diversas razones muchos de ellos optaron por un programa de estudios que se ofreciera en un lugar cercano a su lugar de residencia, y con menores implicaciones económicas en su realización, sin que ello implicara que, en un futuro, fueran a continuar con sus estudios universitarios.

Por otra parte, a los estudiantes encuestados también se les indagó en torno al nivel de escolaridad del padre y de la madre, como elemento que incide en la dinámica socioeconómica del estudiante, a partir del capital cultural que se configura en su dinámica familiar, tanto como de los ingresos económicos que obtiene la familia según el nivel de escolaridad de sus integrantes, para evidenciar, entre los principales resultados, que el nivel de escolaridad tanto del padre como de la madre, se concentra principalmente en el nivel educativo de educación primaria, con unos porcentajes del $65.6 \%$ y $75 \%$ respectivamente. El nivel de escolaridad del padre revela, también, que el $21.9 \%$ tiene una formación de bachillerato y el $12.5 \%$ no posee educación escolar alguna. El nivel de escolaridad de la madre revela que el $15.6 \%$ tiene una formación de bachillerato y el $9.4 \%$ no posee ninguna educación escolar.

Un aspecto que confluye, tanto en los padres como madres de los estudiantes encuestados, radica en que no presentan estudios superiores o universitarios, lo cual genera una dinámica de escaso acceso a capital cultural o de conocimientos especializados en la relación familiar nuclear. Esto puede incidir también en la falta de accesibilidad a información que los hijos e hijas pudieran tener respecto a procesos de Educación Superior, en tanto el acceso a esta dinámica de conocimiento se ve restringida por el escaso conocimiento y/o asesoría que pudieran recibir de sus padres.

Así mismo, la escolaridad del padre y de la madre deja entrever una serie de aspectos, entre los que se destaca, en primera medida, el significativo porcentaje de aquellos que cuentan únicamente con el nivel de educación básica primaria. Al respecto, es mayor el porcentaje de estudiantes quienes cuentan con un padre sin ningún nivel escolar o bachillerato, en comparación con los niveles de escolaridad de la madre. También, se resalta la incidencia de que ninguno de los padres y madres de los encuestados cuenta con un nivel escolar técnico, tecnólogo o profesional, lo que reafirma la caracterización socioeconómica de los estudiantes como una población de escasos recursos económicos que, al residir en una zona rural, dispone 
de un menor porcentaje de probabilidades de ingresar a la Educación Superior universitaria, por lo que encuentra en el ciclo complementario una alternativa académica para la formación como docente.

En este sentido, autores como Bourdieu y Passeron ${ }^{8}$ tratan el tema de las desigualdades en el acceso a la Educación Superior a partir de la división de las esferas o clases sociales. Según los autores en mención, a pesar de que en las democracias actuales la educación se convierte en un derecho fundamental de las personas, sigue siendo selectiva, al depender del origen social y de las capacidades culturales y económicas de quienes desean ingresar. Bourdieu y Passeron examinan las posibilidades de ingreso de diversas esferas sociales a las Facultades universitarias, para plantear que las clases más desfavorecidas tienen un mínimo margen de posibilidades de ingresar a una universidad, en comparación con las clases medias y los sectores superiores o profesionales, lo que determina, de acuerdo con el medio social, una imagen de los estudios superiores como futuro "imposible", "posible" o "normal", que incide en las vocaciones educativas.

Por otra parte, los autores señalan que la elección de las Facultades o Programas de Ciencias y de Letras es una expresión de la desventaja educativa propia de los sujetos de las clases inferiores y medias, "si es verdad que los estudios de ciencias parecen menos ligados al origen social y si se acuerda finalmente que es en la enseñanza humanista donde la influencia del origen social se manifiesta con mayor claridad". ${ }^{9}$ En este sentido, según los planteamientos de los autores, a los Programas y Facultades de formación de maestros, los integran mayoritariamente las clases bajas y medias, quienes encuentran en estos espacios académicos una alternativa de formación ante las desiguales condiciones en las que se compite por un cupo de acceso a la Educación Superior entre aquellos que han tenido mejores condiciones económicas y educativas en la etapa de bachillerato, que se refleja en mejores puntajes de admisión para las carreras más demandadas.

Una vez establecidos algunos de los elementos que caracterizan a la población estudiantil del ciclo de formación complementaria a partir de sus características socioeconómicas, se logra generar una contextualización del

8 Pierre Bourdieu y Jean Passeron, Los herederos, los estudiantes y la cultura (Buenos Aires: Siglo XXI, 2009).

9 Ibíd., 22. 
entorno social y educativo en los procesos de formación de los docentes normalistas del municipio de La Cruz. Ahora, la información obtenida se convierte en insumo para la configuración de los conocimientos, habilidades $\mathrm{y}$ actitudes que se pretenden proponer para la formación de docentes que respondieran a las necesidades de educar en la tolerancia y reconciliación, en la resolución de conflictos, la convivencia y el fortalecimiento del tejido social, consecuente con las necesidades regionales en materia de construcción y promoción de una cultura de paz.

\section{Avances y retos en educación para la paz en la Escuela Normal Superior del Mayo}

Uno de los interrogantes que se plantea en este acápite gira en torno a la pregunta: ¿qué aspectos en educación para la paz se han implementado en el ciclo de formación complementaria de la Escuela Normal Superior de La Cruz - Nariño? Para ello, se realizó una serie de entrevistas a docentes de la institución educativa en estudio, con el objetivo de obtener información acerca de las estrategias de enseñanza, proyectos educativos y procesos de aprendizaje que se vienen implementando en el ciclo de formación complementaria respecto a los elementos que componen la educación para la paz. En este sentido, se enfatizó en los proyectos pedagógicos transversales que se adelantan en la institución, la incorporación de la cátedra de paz en los Planes de estudio y la formación de los docentes normalistas en materia de educación para la paz en el ciclo de formación complementaria.

Entre los resultados de la indagación realizada, encontramos que, en la institución educativa en estudio, la educación para la paz, entendida como los procesos formativos en tolerancia, reconciliación, resolución de conflictos, convivencia, fortalecimiento del tejido social, democracia y desarrollo sostenible, se implementa de manera transversal, a partir de cuatro ámbitos como el proyecto pedagógico en educación para la paz y la democracia, los contenidos curriculares de las áreas de Ciencias Sociales y Ética y Valores, el Comité de Convivencia Escolar y los seminarios de competencias ciudadanas y psicología social, que se imparten en el ciclo de formación complementaria. Desde estos ámbitos, la Escuela Normal Superior de La Cruz ha venido desarrollando una serie de componentes educativos, desde los cuales se pretende aportar al fortalecimiento de una cultura de paz entre la comunidad educativa y el entorno que rodea a esta institución. 
Al respecto, el primero de estos ámbitos gira en torno al proyecto pedagógico en educación para la paz y la democracia, que se implementa en todos los grados de la institución educativa y que tiene como propósito fomentar una cultura democrática a partir de la elección de representantes estudiantiles, desde donde se generan procesos de debate académico, respeto por las diferencias ideológicas, propuestas factibles ante las necesidades escolares, entre otros aspectos. Este proyecto se desarrolla con mayor énfasis al inicio del año académico, mediante la elección del gobierno escolar, proceso desde el cual los estudiantes comparten y argumentan sus propuestas para el mejoramiento de la convivencia, la construcción de soluciones ante las problemáticas escolares y la defensa de los derechos estudiantiles. Al respecto, algunos de los docentes manifiestan:

En la institución se viene realizando el proyecto de educación para la paz, que gira en torno, principalmente, a la elección del gobierno escolar. Dentro de la elección de los representantes estudiantiles, se hace todo un proceso democrático o de sensibilización, porque creemos y tenemos la esperanza de que con este proyecto tratemos de inculcar a nuestros estudiantes una sana práctica de la política, de que no volvamos a caer en el clientelismo político, la compra de votos, la manipulación mediática, sino que todo sea un proceso político muy claro, transparente, mediante la elección del personero estudiantil, para hacer un trabajo como cuando uno trata de capturar votos como hacen los políticos, pero acá con ideas y, por eso, la elección del gobierno escolar es un proceso que fortalece la democracia, la tolerancia, la ética, la honestidad entre los estudiantes y la comunidad educativa. ${ }^{10}$

En el proyecto de educación para la paz y la democracia se vienen trabajando temas, incluyendo los de gobierno escolar, democracia y formas de participación democrática, derechos humanos, derechos del niño; se inculcan la parte de valores, valores cívicos, valores ciudadanos y haciendo énfasis en la cultura ciudadana de toda la comunidad educativa, padres de familia, estudiantes y docentes. Este es un proyecto no solo destinado a los estudiantes de formación complementaria, sino a toda la institución en general, donde el área de Ciencias Sociales es la que organiza los parámetros y se los desarrolla en los distintos cursos. ${ }^{11}$

Así, el proyecto pedagógico en educación para la paz y la democracia se convierte en un referente pedagógico para la institución educativa en materia

\footnotetext{
${ }^{10}$ Hernán Bedoya, docente de la Escuela Normal Superior de La Cruz, en entrevista con el autor, abril de 2018 .

${ }^{11}$ Jorge Viveros, docente de la Escuela Normal Superior de La Cruz, en entrevista con el autor, abril de 2018.
} 
de educación para la paz. Este proyecto se encamina principalmente en los procesos de elección de representantes estudiantiles y en la formación en valores cívicos y cultura ciudadana en los diferentes niveles educativos que ofrece la institución, razón por la cual es importante reconocer su aporte al fortalecimiento de una cultura de paz y de la democracia; sin embargo, la formación específica que requieren los docentes normalistas en educación para la paz, no puede fundamentarse únicamente en el proyecto pedagógico que se desarrolla en la institución, puesto que su formación como docentes requiere el fortalecimiento de unas competencias específicas, para el pertinente ejercicio de su profesión en los diferentes contextos que lo requieran.

Al examinar el segundo ámbito que se viene desarrollando en la Escuela Normal Superior del Mayo, sobre educación para la paz, los docentes se refieren a los contenidos curriculares de las áreas de Ciencias Sociales y Ética y Valores, desde las cuales se estudian procesos sociales, como el conflicto armado en Colombia y su incidencia en la región, la formación en derechos humanos, valores ciudadanos y demás aspectos que intervienen en los procesos formativos de los estudiantes desde el campo de las Ciencias Sociales. Al respecto, algunos de los docentes manifiestan:

En el área de Ciencias Sociales se trabaja el tema del conflicto y el posconflicto; de acuerdo al grado, se trata de incluir temáticas para hacerles conocer desde cuándo ha comenzado este conflicto interno en Colombia, cómo ha sido su historia, cómo ha sido su evolución y también mirar las posibilidades que nos dan hoy en día los nuevos sistemas políticos en cuanto a la paz y relacionarlos a ellos con ese entorno. ${ }^{12}$

Dentro del área de Ciencias Sociales, se está empezando a trabajar lo de la cátedra de paz. Con los niños, intentamos que ese tema no sea simplemente tablero o cuaderno, sino que sea vivencial, y eso me parece importante que los futuros docentes lo tengan en cuenta, que la cátedra de paz no caiga en un academicismo, sino que se convierta en un espacio para el análisis de la sociedad, de nuestros conflictos y desde donde podamos proponer soluciones a las problemáticas inmediatas. $^{13}$

${ }^{12}$ Jairo Narváez, docente de la Escuela Normal Superior de La Cruz, en entrevista con el autor, abril de 2018.

${ }^{13}$ Saúl López, docente de la Escuela Normal Superior de La Cruz, en entrevista con el autor, abril de 2018. 
Según los Lineamientos curriculares emanados por el Ministerio de Educación Nacional, en la cátedra para la paz se desarrollan ejes temáticos relacionados con el surgimiento del conflicto armado, los procesos históricos de violencia y las alternativas de paz que en el tiempo se han intentado materializar para la finalización de la guerra. Estos aspectos, que se tratan a lo largo de los diferentes niveles de la educación primaria y secundaria, son el escenario propicio en el cual se pretende implementar la cátedra de la paz como un espacio para fomentar el proceso de apropiación de conocimientos y competencias relacionados con el territorio, la cultura, el contexto social y la memoria histórica, tal como se plantea en el Decreto 1038 , de 2015.

El tercerámbito de análisis gira en torno al Comité de convivencia escolar, que se presenta en la institución, desde el cual se tratan problemáticas que afectan las relaciones interpersonales y de convivencia entre la comunidad educativa. Este Comité lo integran el rector de la institución, el personero estudiantil, el psicólogo orientador, el coordinador de convivencia, el representante del Consejo de padres de familia, un representante del consejo estudiantil y un representante docente. Su principal objetivo es orientar y coordinar estrategias, programas y actividades para la prevención y mitigación de la violencia escolar y formación para los derechos humanos, sexuales y reproductivos de los alumnos del plantel, en el marco de la corresponsabilidad de los miembros de comunidad educativa que presiden.

Así, desde este órgano institucional, se propicia por intervenir los conflictos que se pueden presentar entre quienes integran la comunidad educativa, además de liderar acciones que fomentasen la convivencia y la cultura de paz en el contexto escolar. Por tanto, es pertinente resaltar el papel que cumple este organismo en la prevención y atención de acontecimientos que afectan el bienestar psicosocial de los educandos, con atención, de manera oportuna, a las situaciones de conflicto, las conductas de violencia o de vulneración de derechos que afectan el ambiente escolar de la institución educativa, como parte del entorno social del municipio de La Cruz.

Por último, el cuarto ámbito se centra específicamente en los procesos que se adelantan en el ciclo de formación complementaria, en materia de educación para la paz. Aunque si bien los tres ámbitos referenciados antes incluyen a los estudiantes del ciclo complementario como parte de la Escuela Normal Superior del Mayo, es pertinente examinar las acciones dirigidas 
específicamente a los maestros en formación para el fortalecimiento de los aspectos relacionados con la educación para la paz, la resolución de conflictos, la prevención del acoso escolar y la participación democrática. En este sentido, los docentes entrevistados manifiestan:

En el ciclo de formación complementaria se hace un trabajo que se ha venido desarrollando poco a poco desde el área de competencias ciudadanas, que se implementó en el tercer semestre del programa; se trabaja con ellos algunas estrategias y algunos mecanismos sobre competencias ciudadanas, como, por ejemplo, toda la parte de los valores que se deben enseñar desde la primaria, y cómo los niños y niñas deben aprender haciendo, con ejercicios prácticos, cotidianos, para que ellos los puedan asimilar para su vida. ${ }^{14}$

En el ciclo complementario, se trabaja la práctica pedagógica integral e investigativa, la cual es un espacio para reflexionar sobre el actuar del maestro. Se trabaja lo de cátedra de paz desde las problemáticas que tiene el país, pero digamos que muy explícito no ha estado lo de educación para la paz, sino que, desde las diferentes áreas, de forma transversal, se han venido haciendo unos esfuerzos por tratar de incorporarla en nuestros lineamientos curriculares, pero sí considero que habría que trabajarla en profundidad..$^{15}$

Ante los elementos señalados por los docentes, se logran identificar los esfuerzos por fortalecer la formación en educación para la paz desde las competencias ciudadanas, la psicología social y la práctica pedagógica integral e investigativa. Desde el área de competencias ciudadanas, que se imparte en el ciclo complementario, se tratan los ejes temáticos relacionados con la formación ciudadana de los estudiantes de básica primaria, desde los aspectos cognitivos, procedimentales y comportamentales. Desde el área de psicología social, los docentes en formación identifican los procesos cognitivos de los estudiantes, así como, también, examinan los diferentes aspectos sociales que inciden en los procesos de enseñanza y aprendizaje $\mathrm{y}$, por último, desde su práctica pedagógica, logran integrar los diferentes elementos trabajados en su formación, para evaluar, reflexionar y optimizar su ejercicio docente.

Así, se logran identificar los aspectos que, en educación para la paz, se han implementado en el ciclo de formación complementaria de la Escuela

${ }^{14}$ Jorge Viveros, docente de la Escuela Normal Superior de La Cruz, en entrevista con el autor, abril de 2018.

${ }^{15}$ Oliden Muñoz, docente de la Escuela Normal Superior de La Cruz, en entrevista con el autor, abril de 2018. 
Normal Superior de La Cruz - Nariño. Los esfuerzos por fortalecer la cultura de paz son visibles a través de la implementación del proyecto pedagógico en educación para la paz y la democracia, los contenidos curriculares de las áreas de Ciencias Sociales y Ética y Valores, el Comité de convivencia escolar y los seminarios de competencias ciudadanas, psicología social y la práctica pedagógica integral e investigativa, que se imparte en el ciclo de formación complementaria.

Sin embargo, es menester señalar que los aspectos didácticos y la formación en competencias docentes para desarrollar los procesos de enseñanza sobre la cátedra de paz, no se tratan con mayor especificidad en el ciclo de formación complementaria. Por esta razón, esta investigación plantea la necesidad de establecer ejes de fortalecimiento en competencias didácticas en educación para la paz, en instituciones educativas que, como la Escuela Normal Superior de La Cruz, han afrontado las secuelas del conflicto armado y la etapa del postconflicto a través de la formación de educadores que respondieran a los requerimientos de transformación social que requiere su entorno.

\section{Competencias docentes en educación para la paz: aportes en la formación de maestros en el municipio de La Cruz}

En este último apartado, se pretende examinar la forma en que las experiencias previas en educación para la paz, desarrolladas en la Escuela Normal Superior de La Cruz, pueden ampliarse y fortalecerse a partir de los planteamientos conceptuales y metodológicos que se referencian en la literatura académica que gira alrededor de la temática de estudio, para proponer un ejercicio analítico de doble vía, en tanto se plantean alternativas pedagógicas para el quehacer docente desde referentes académicos, mientras que, desde la experiencia empírica, se nutren los análisis y enfoques que pueden establecerse en el marco conceptual de la educación para la paz.

El primer elemento que se logra destacar en torno a la formación de docentes, en el municipio de La Cruz, es la necesidad de que los futuros maestros reconocieran los territorios afectados por el conflicto como espacios de requerimientos pedagógicos diferenciales; es decir, al tomar en cuenta que la Escuela Normal Superior del Mayo forma docentes para la educación preescolar y básica primaria, se requiere reflexionar en torno a las características que se presentan en las escuelas ubicadas en contextos 
urbanos y rurales, aquellas a las que ha afectado el conflicto, para así reconocer una serie de particularidades que diferencian, además de sus modos de vida, sus estilos de enseñanza y aprendizaje.

Entonces, se debe partir del reconocimiento de la institución educativa y su contexto, y de la necesidad de fortalecer un enfoque para la paz en los procesos educativos que el docente lidera. Al tomar en cuenta que a las condiciones de dificultad que caracterizan a las escuelas rurales las antecede la falta de apoyo estatal tanto en el mejoramiento de la planta física como en el bienestar cotidiano de los estudiantes y las condiciones laborales de los educadores. Sumado a esto, las políticas educativas de formación de maestros se desarrollan a partir de contenidos desarticulados con los ambientes de violencia y conflicto que se viven en Colombia; por tanto, la voluntad del docente en la autoformación y el continuo mejoramiento de su práctica profesional es el esfuerzo que requiere cada uno de los contextos educativos con necesidades específicas en materia de educación para la paz.

Las reflexiones que han realizado los docentes del municipio de La Cruz sustentan la necesidad de una educación que eliminase todos los factores de exclusión dentro y fuera de las aulas, que fomentase el diálogo, el respeto por las diferencias, la participación de los estudiantes en los espacios democráticos, y que contribuyeran a la transformación social de su entorno y al mejoramiento de las condiciones de vida de sus familias. Así mismo, los docentes encargados de la formación de educadores normalistas señalan la importancia de construir una escuela alternativa, que se conecte con los territorios para construir comunidad y aportar a la cultura de paz, puesto que se crítica la actual desconexión que existe entre las escuelas y su contexto social, lo que convierte a las instituciones educativas en burbujas académicas, que no responden ni se conectan con la realidad y las necesidades sociales del entorno.

Un segundo elemento de reflexión que surge de la literatura académica hacia las dinámicas educativas en la formación de docentes tiene que ver con las propuestas curriculares en educación para la paz que se plantean desde autores como Sara Victoria Alvarado, ${ }^{16}$ para quien la construcción de paz desde las escuelas no solo debe contar con un interés específico de

${ }^{16}$ Sara Victoria Alvarado, "Ciudadanías y competencias ciudadanas", Estudios políticos Vol. 40. No. 1, (2012): 53 - 75. 
los docentes, sino, también, debe respaldarla una propuesta curricular que resultara acorde con los objetivos misionales de la institución. Por tanto, uno de los llamados que se genera desde la literatura académica se relaciona con insertar en los procesos curriculares aspectos como la enseñanza de lo propio, donde los contenidos de la escuela se desarrollaran con el medio local en el cual los estudiantes desarrollan su vida, para buscar la generación de una apropiación de los contenidos temáticos a partir de la interacción de los estudiantes con el entorno que los rodea.

Así mismo, se plantea la pertinencia de entender los espacios escolares como un microcosmos desde donde se pueden plantear alternativas de paz ante las problemáticas de violencia que acontecen en las escuelas, que reflejan las dinámicas del mundo que los rodea. En este ámbito, la resolución de conflictos, la participación en la toma de decisiones y el mejoramiento en las relaciones de convivencia que se configuran entre los estudiantes pueden, a largo plazo, articularse con las formas de actuar que tuviera el estudiante con un entorno más amplio, para establecer una ruta de aprendizaje que surge en los espacios escolares, pero que se amplía y se aplica en los entornos de vida de los estudiantes.

Otro de los aspectos a establecer son las relaciones comunicativas entre la escuela, la familia y el entorno, desde un punto en el cual el diálogo permitiera articular los lazos sociales y familiares entre la comunidad educativa. En este sentido, se plantea la importancia de articular a los padres y madres de los estudiantes como formadores fundamentales de los niños, ya que la cultura de paz asienta sus bases en la sociabilidad y el diálogo entre los integrantes de la familia. Por tanto, la labor docente parte de la constante comunicación con la familia y la vinculación de los procesos de aprendizaje con el entorno cercano que sitúa y rodea a los estudiantes.

Un tercer elemento para tener en cuenta pretende reflexionar en torno a la formación que reciben los docentes normalistas, no solo a través de los proyectos pedagógicos que implementa la institución en todos los niveles educativos, sino, también, a partir de las competencias específicas que se pretenden desarrollar en los maestros en el ciclo de formación complementaria. Esta situación surge a partir de examinar que los esfuerzos realizados por la Escuela Normal Superior del Mayo, en materia de educación para la paz, se desarrollan a través de los contenidos curriculares de las asignaturas de Ética y Valores y Ciencias Sociales, que se imparten en la institución, y el proyecto en educación para la paz y la 
democracia, en el cual se seleccionan los representantes estudiantiles y se crean espacios de diálogo en la comunidad educativa; sin embargo, resulta pertinente que los educadores en formación tuvieran las competencias necesarias no solo para formar parte de estos procesos, sino, además, que ellos los organizaran y lideraran a partir de su futuro rol como docentes en los niveles de preescolar y básica primaria.

De esta forma, las competencias necesarias para liderar proyectos pedagógicos en materia de paz requieren de los docentes una serie de capacidades para conducir al estudiantado hacia la consecución de objetivos específicos, tanto en competencias básicas como la habilidad de interpretar y proponer soluciones a las problemáticas de la vida cotidiana de su entorno, así como en competencias genéricas y específicas, que le permita proyectar el desarrollo y ejecución de proyectos educativos, el tratamiento ético y reflexivo de las situaciones de conflicto en el ambiente escolar y el desarrollo pedagógico de los lineamientos planteados en la cátedra para la paz.

Como cuarto elemento de reflexión, se destacan los aportes de autores como Paulo Freire (1972), para quien las prácticas del sistema tradicional de la educación (memorístico, competitivo y cartesiano) se reconocen como prácticas educativas "bancarias", donde solo se deposita la información en la cabeza vacía del educando. Esta crítica al sistema educativo tradicional hace un llamado a las instituciones y docentes a insertar la educación para la paz en modelos pedagógicos alternativos, que se fundan no solo en la razón, sino, también, en las emociones y fomentan "el desarrollo de experiencias vivenciales que conduzcan a intuir la paz como modo de ser de las relaciones interpersonales". ${ }^{17}$

A partir de lo enunciado, entre los aspectos, tanto cognitivos como emocionales y éticos, que conducen al docente a liderar procesos en educación para la paz, Jaime Parra postula una serie de características, entre las que se destacan:

1. Ser capaces de utilizar los sentidos, la imaginación, el pensamiento y el razonamiento, de un modo verdaderamente humano, de una manera informada y cultivada por la imaginación y el pensamiento para la experimentación y la producción de obras y actos literarios, musicales, etc.

${ }_{17}$ Felipe MacGregor, Cultura de paz. (Lima: Asociación Peruana de Estudios para la Paz, 1986). 
2. Ser capaces de tener vínculos afectivos con otras personas y con los objetos del mundo. Promover esta capacidad significa también crear modos solidarios de asociación entre las personas.

3. Hace alusión a la relación con los otros seres humanos en una adecuada condición de desarrollo personal. La afiliación presenta una dimensión social y una personal. La dimensión social hace referencia a ser capaz de convivir con otros y en función de los demás, reconocer y mostrar interés por los otros seres humanos y comprometerse en diversas formas de interacción social.

4. Ser capaces de vivir una relación cercana y con preocupación por el bienestar de los animales, las plantas y el mundo natural.

5. Esta capacidad hace alusión a la dimensión política, es decir, ser capaces de participar efectivamente en las decisiones políticas que gobiernan nuestra vida; tener derecho a la participación política y a la protección de la libertad de expresión y de asociación. ${ }^{18}$

Estos principios responden a las competencias que requieren los docentes y en torno a las cuales se pretende formar a los estudiantes en aspectos como la comunicación asertiva, el uso de estrategias comunicativas para la resolución de conflictos escolares, la generación de ambientes de convivencia, el manejo de las emociones, entre otros aspectos cognitivos y metodológicos que propone el Ministerio de Educación Nacional en la cátedra para la paz. Estos elementos pretenden preparar a los niños de los niveles de preescolar y básica primaria para la generación de alternativas de paz, a través de ejercicios de pensamiento reflexivo.

Al respecto, cabe señalar que los horizontes hacia donde se construyera la educación para la paz van muy ligados al tipo de paz y de sociedad que proyectan los gobiernos y las instituciones. Por ello, el interés por la construcción de una cultura de paz desde las regiones afectadas por el conflicto debe ser el elemento que orientase las decisiones gubernamentales, especialmente porque políticas como la implementación de la cátedra de paz requieren establecerse desde el diario vivir de las poblaciones y no solo desde las directrices estatales. Por ello, flexibilizar el estudio de la paz, no solo como una asignatura independiente, sino como un aspecto que atraviesa toda la estructura y vida escolar, es decir, como un principio educativo, que posibilitará a los docentes llevar los contenidos de la cátedra para la paz, más allá de un discurso coyuntural, a una reflexión ligada a las experiencias y realidades de la vida cotidiana de los estudiantes.

${ }_{18}$ Jaime Parra, El desarrollo de las capacidades docentes. (Bogotá: Instituto para la Investigación Educativa y el Desarrollo Pedagógico, 2013), 24. 
En el último aspecto, cabe señalar los retos de las instituciones educativas formadoras de docentes, que implican reflexiones estructurales, curriculares, éticas y pedagógicas, acordes a las necesidades sociales y educativas del contexto colombiano. El rol de los docentes, tanto de los espacios urbanos como rurales, es fundamental para la construcción de una cultura de paz en el país, puesto que están en permanente contacto con aquellos territorios donde el olvido del Estado, la desigualdad social, la incidencia de los grupos armados y de los cultivos ilícitos, influyen los proyectos de vida de los niños y jóvenes, que encuentran en la educación una posibilidad de construir un mejor futuro para su vida y un bienestar integral para su familia; de ahí que reflexionar en los procesos de formación de los futuros docentes, desde el municipio de La Cruz, se torna pertinente no solo para las dinámicas de esta región del Departamento de Nariño, sino, también, para todas aquellas regiones que conciben a la paz como algo más que el silenciamiento de las armas; también, como la generación de oportunidades de vida a partir de la educación, la salud, el trabajo, el desarrollo humano y la cultura de la población colombiana.

Para finalizar, entonces, la propuesta presentada en este texto se sintetiza en cinco aspectos, que permiten fortalecer los procesos de formación docente en educación para la paz, que se configuran desde las realidades presenciadas en la formación de maestros normalistas en el municipio de La Cruz y los lineamientos conceptuales que diversos autores han trabajado en torno a procesos de educación, paz y formación de educadores y educandos en las últimas décadas. El primero, gira en torno a la pertinencia de indagar por la dinámica social e histórica de los territorios en los que se sitúan las instituciones educativas y, por ende, los procesos de aprendizaje. El segundo elemento versa sobre la necesidad de enfocar las propuestas curriculares con los procesos sociales y culturales de construcción de paz. El tercer eje se plantea a partir de la importancia de que los maestros configurasen un proyecto de vida en torno a la docencia, para así tener una comprensión de las prácticas pedagógicas, no solo como una dinámica instrumentalizada de compartir conocimiento, sino de construcción de proyectos de vida de las nuevas generaciones. El cuarto aspecto retoma la necesidad de educar para la vida, a partir de proyectos que fueran más allá de las aulas y los contenidos académicos y, sobre todo, que fomentasen la conciencia del ser social de las personas. Y el último elemento, que enfatiza en la necesidad de asumir y transformar 
los retos y necesidades de las dinámicas educativas, campo en el que inciden problemas como la violencia, la pobreza, las desigualdades, el olvido, las precarias condiciones de bienestar, que inciden en el quehacer pedagógico de los maestros.

\section{Conclusiones}

En primera instancia, se reconoce el papel de las instituciones educativas en la satisfacción de las necesidades y requerimientos del saber en la población, que le permitiera comprender el mundo que la rodea y proponer actitudes y comportamientos pertinentes con el desenvolvimiento social, cultural, económico y político de los individuos. Por tanto, el papel del docente en los procesos de enseñanza requiere una actualización continua ante los cambios de la sociedad y las realidades específicas de los territorios, para tratar las crecientes necesidades de los estudiantes.

A partir de lo expuesto, los educandos del ciclo de formación complementaria de las Escuelas Normales Superiores fundamentan su formación en la identificación de las capacidades, intereses, ritmos, motivaciones y experiencias diferentes que caracterizan los procesos de aprendizaje de los estudiantes, en especial de aquellos que se encuentran en la etapa de preescolar y básica primaria. Por ello, entre los propósitos de formación de los maestros normalistas, se encuentra la aceptación y respeto a las diferencias y la diversidad, la inclusión de las capacidades múltiples de aprendizaje y la relación de los contenidos del saber con el entorno social y natural que los rodea, para fomentar la reflexión y el compromiso de las personas en la construcción de un bienestar para la población.

Respecto al fortalecimiento de las capacidades investigativas dentro de su práctica pedagógica, se establece fomentar tanto el estudio de las vivencias en los contextos educativos como, también, el quehacer pedagógico de los docentes, con el objetivo de reflexionar e indagar en torno a las problemáticas educativas y pedagógicas, para propender por el mejoramiento de las relaciones y las acciones que acontecen en las instituciones, mediante proyectos pedagógicos e investigativos que, como se logró dar cuenta en la población de estudio, posibilitan la creación de espacios de aprendizaje y convivencia a través de mecanismos alternativos de resolución de conflictos, formación y empoderamiento de líderes 
estudiantiles, creación de estrategias de uso del tiempo libre, entre otros aspectos, configurados a partir del trabajo en equipo, desde los diferentes actores que conforman la comunidad educativa.

Otro de los aspectos a considerar tiene que ver con las experiencias y prácticas que el docente puede desarrollar en materia de educación para la paz desde los contenidos curriculares de las diferentes áreas de conocimiento, puesto que educar para la paz no es una responsabilidad específica de una asignatura o un docente, sino se construye a partir de las habilidades de pensamiento, los Planes de estudio y las metodologías pedagógicas que contribuyeran a la formación integral de las personas, si se consideran los contextos sociales y culturales y las características propias de las etapas de desarrollo de los estudiantes. Por ello, se concluye que las propuestas curriculares desde las cuales se forman los maestros normalistas deben establecer vínculos entre los aspectos que se pretenden alcanzar desde los modelos pedagógicos y las realidades escolares que requieren conocimientos prácticos y que permiten atender las problemáticas y preguntas cotidianas de los niños de la educación preescolar y básica primaria, para construir formas de llegar al conocimiento a partir del entorno que los rodea.

Además, entre las capacidades a fortalecer, se destaca la reflexión del maestro sobre sus propias prácticas educativas y la relación con el entorno; es decir, la capacidad de reflexionar, cuestionar y mejorar su quehacer docente a través del análisis de los fundamentos que orientan su enseñanza, los recursos pedagógicos que utiliza y las estrategias que desarrolla en su práctica educativa. De esta manera, mediante la exploración de sus propias prácticas, los docentes podrán articular de mejor manera sus actividades profesionales con las necesidades y requerimientos de la comunidad educativa donde se encuentran inmersos, para coadyuvar al fortalecimiento de los tejidos sociales, económicos y culturales entre las instituciones educativas y la población.

Los ámbitos de fortalecimiento en la formación de docentes normalistas ya planteados se suman a los esfuerzos que se vienen realizando en la Escuela Normal Superior del Mayo, en el Proyecto pedagógico de educación para la paz y la democracia, los contenidos curriculares de las áreas de Ciencias Sociales y Ética y Valores, el Comité de convivencia escolar y los seminarios de competencias ciudadanas y psicología social, que se imparten en el ciclo de formación complementaria. 
Así mismo, se han planteado conocimientos, habilidades y actitudes que requieren los maestros para una educación orientada hacia la construcción de paz desde los contextos escolares, a partir de ejes transversales, como la responsabilidad social en el ejercicio de la profesión docente, la formación de su identidad y de sus valores éticos, la voluntad de comprometerse en los procesos sociales de su entorno y el respeto a las diferencias culturales que puedan presentarse en las dinámicas educativas. Así, las competencias que se deben fortalecer pretenden generar en el docente unas bases de sentido humano, de compromiso social y de capacidad reflexiva, que orientan su praxis profesional.

Por último, para afianzar los principios pedagógicos sobre los cuales se fundamenta la formación de docentes normalistas, se logran establecer unas áreas de fortalecimiento en materia de competencias docentes en educación para la paz, que posibilitará a los maestros atender de forma oportuna las necesidades pedagógicas en materia de derechos humanos, resolución de conflictos, prevención del acoso escolar y participación ciudadana, que les posibiliten responder a las necesidades de educar en la tolerancia y reconciliación, en la resolución de conflictos, la convivencia y el fortalecimiento del tejido social, consecuente con el entorno que los rodea. Por ello, el mejoramiento de capacidades, como educar en la no violencia, la convivencia en los contextos educativos y la resolución pacífica de conflictos, se convierten en los pilares fundamentales que contribuyen a configurar territorios de paz, mediante el fortalecimiento de la formación de los maestros en los territorios con mayor incidencia del conflicto armado en Colombia.

\section{Referencias}

Alvarado, Sara Victoria. "Ciudadanías y competencias ciudadanas". Estudios políticos, vol. 40. No. 1, (2012): 53-75.

Bedoya, Hernán. Docente de la Escuela Normal Superior de La Cruz, en entrevista con el autor, abril de 2018.

Bourdieu, Pierre y Passeron, Jean. Los herederos, los estudiantes y la cultura. Buenos Aires: Siglo XXI, 2009.

Bruner, Jerome. La educación, puerta de la cultura. Madrid: Visor, 1997. 
Freire, Paulo. Pedagogía del oprimido. Buenos Aires: Tierra Nueva/Siglo XXI, 1972.

López, Saúl. Docente de la Escuela Normal Superior de La Cruz, en entrevista con el autor, abril de 2018.

MacGregor, Felipe. Cultura de paz. Lima: Asociación Peruana de Estudios para la Paz, 1986.

Mora-García, José. “Aproximación a las Pedagogías Alternativas: de la Pedagogía de la Diversidad a las Pedagogías de la Resiliencia en el Marco del Postacuerdo". Revista Historia de la Educación Colombiana. Vol. 22 No 22, (2019): 39-66. https://doi.org/10.22267/rhec.192222.51.

Muñoz, Oliden. Docente de la Escuela Normal Superior de La Cruz, en entrevista con el autor, abril de 2018.

Muñoz Bravo, José. "Las Escuelas Normales, orientan la formación de maestros superiores, comprometidas con la educación y la práctica pedagógica investigativa". Revista Historia de la Educación Colombiana vol. 23 No 23 (2019), 139-171. https://doi.org/10.22267/rhec.192323.62.

Narváez, Jairo. Docente de la Escuela Normal Superior de La Cruz, en entrevista con el autor, abril de 2018.

Parra, Jaime. El desarrollo de las capacidades docentes. Bogotá: Instituto para la Investigación Educativa y el Desarrollo Pedagógico, 2013.

Pinilla, Análida. Reflexiones en educación universitaria. Bogotá: Unibiblios, 1999.

Presidencia de la República de Colombia. Decreto 1038 de 2015 (mayo 25), https:// diario-oficial.vlex.com.co/vid/decreto-numero-1038-2015-571203254

Restrepo Mesa, Karol. "Propuesta de Formación del Maestro-Mediador para la Consolidación de la Cultura de Paz en Colombia". Revista Historia de la Educación Colombiana. vol. 22 No 22, (2019): 67-91. https://doi. org/10.22267/rhec.192222.52.

Viveros, Jorge. Docente de la Escuela Normal Superior de La Cruz, en entrevista con el autor, abril de 2018. 


\section{Citar este artículo}

Quintero Timaná, Diego Andrés. "Formación docente en educación para la paz, en la Escuela Normal Superior del municipio de La Cruz - Nariño". Revista Historia de la Educación Colombiana. Vol. 24 No 24 (2020): 77-103

DOI: https://doi.org/10.22267/rhec. 202424.73 